\title{
Comparison of 3D Flow Fields Arising in Mixed and Standard Unstructured Finite Elements
}

\author{
Annamaria Mazzia ${ }^{1}$, Giorgio Pini ${ }^{1}$, Mario Putti $^{1}$, and Flavio Sartoretto ${ }^{2}$ \\ 1 Dipartimento di Metodi e Modelli Matematici per le Scienze Applicate, \\ Università di Padova, via Belzoni 735131 Padova, Italy \\ \{mazzia, pini, putti\}@dmsa.unipd.it, http://www.dmsa.unipd.it \\ 2 Dipartimento di Informatica, Università di Venezia, \\ via Torino 155, 30173 Mestre VE, Italy \\ sartoret@dsi.unive.it, http://www.dsi.unive.it
}

\begin{abstract}
Computing 3D velocity fields is an important task in subsurface water flow analysis. While Finite Element (FE) simulations generally yields accurate estimates of the head, the numerical velocity may display errors and even unphysical behavior in some parts of the domain. Theoretically, the Mixed Hybrid FE (MHFE) approach provide a more accurate approximation to the velocities.

In this communication we analyze a number of 3D-flow test cases, and compare the results obtained using $\mathrm{FE}$ and MHFE on tetrahedral meshes. Theoretical convergence estimates are numerically verified for a few simple problems. A more complex heterogeneous test case is used to show that, even for very fine meshes, the results obtained using the two discretization approaches may differ.
\end{abstract}

\section{Introduction}

The Finite Element (FE) and the Mixed Hybrid Finite Element (MHFE) schemes provide numerical solutions to three-dimensional flow equations. They display different behaviors, both for the potential and the velocity fields. In this communication we analyze the characteristics of these two numerical approaches, detailing the emerging differences and the advantages and disadvantages of one method over the other.

The equations governing steady-state 3D porous media flow are:

$$
\left\{\begin{array}{l}
\boldsymbol{\nabla} \cdot \boldsymbol{v}=f \\
\boldsymbol{v}=-K \boldsymbol{\nabla} \psi,
\end{array}\right.
$$

where $\psi$ is the pressure head, $\boldsymbol{v}$ is the Darcy velocity vector, $K$ is the permeability tensor and $f$ is a source or sink term.

Dirichlet and Neumann boundary conditions must be given to identify a well posed mathematical formulation of the flow problem. These conditions are

$$
\begin{aligned}
& \psi(\boldsymbol{x})=\psi_{P}(\boldsymbol{x}) \quad \text { on } \Gamma_{1}, \\
& \boldsymbol{v} \cdot \boldsymbol{n}=-q_{N}(\boldsymbol{x}) \quad \text { on } \Gamma_{2},
\end{aligned}
$$


where $\boldsymbol{x}=(x, y, z)^{T}$ is the Cartesian spatial coordinate vector, $\psi_{P}$ is the prescribed pressure head (Dirichlet conditions) on the boundary portion $\Gamma_{1}, \boldsymbol{n}$ is the outward normal unit vector, and $q_{N}$ is the prescribed flux (Neumann condition) across the boundary portion $\Gamma_{2}$. We adopt the convention of considering the flux, $q_{N}$, positive when it is inward, negative when outward.

\subsection{FE and MHFE Solution}

Let $T_{l}$ be a generic tetrahedron of the three-dimensional triangulation discretizing the domain, and let $p$ be the total number of nodes, let $m$ be the total number of elements, and $n$ the total number of faces (triangles). This computational mesh is defined for both the FE and MHFE schemes.

The FE method considered in this paper is a standard Galerkin approach that employs linear basis functions for the pressure head at the nodes:

$$
\psi \approx \sum_{i=1}^{p} \psi_{i} N_{i}(x, y, z)
$$

where $N_{i}(x, y, z)=a_{i} x+b_{i} y+c_{i} z+d_{i}$. Velocities are proportional to the gradient of the pressure, which in this case is constant over each tetrahedron. They can be defined as:

$$
\boldsymbol{v}^{(l)}=-K\left[\begin{array}{c}
a_{i}+a_{j}+a_{k}+a_{m} \\
b_{i}+b_{j}+b_{k}+b_{m} \\
c_{i}+c_{j}+c_{k}+c_{m}
\end{array}\right],
$$

where $i, j, k, m$ denote the four nodes that define tetrahedron $T_{l}$. This approach is the natural method for evaluating velocities in $\mathrm{FE}$ schemes and is also the most accurate when no additional post processing is exploited. It is known that the Galerkin approach on tetrahedrons may lead to numerical inaccurate and not mass-conserving fluxes, in particular for heterogeneous conductivity tensors [1, 23].

The MHFE formulation that we exploited is an extension to 3D tetrahedral meshes of a $2 \mathrm{D}$ algorithm for triangular meshes [4.5]. The pressure $\psi$, the Lagrange multipliers $\lambda$, and the flux $\boldsymbol{v}$ are approximated by

$$
\psi \approx \sum_{j=1}^{m} \psi_{j} \phi_{j}, \quad \lambda \approx \sum_{j=1}^{n} \lambda_{j} \mu_{j}, \quad \boldsymbol{v} \approx \sum_{j=1}^{n} v_{j}^{(l)} \boldsymbol{w}_{j}^{(l)},
$$

where $\phi_{j}$ and $\mu_{j}$ are $P_{0}$ basis functions, and $\boldsymbol{w}_{j}^{(l)}$ are the discontinuous RaviartThomas [67] vector basis functions. The final system of algebraic equations is:

$$
\left(\begin{array}{ccc}
A & -B & Q \\
B^{T} & 0 & 0 \\
Q^{T} & 0 & 0
\end{array}\right)\left(\begin{array}{c}
\mathbf{v} \\
\boldsymbol{\psi} \\
\boldsymbol{\lambda}
\end{array}\right)=\left(\begin{array}{c}
0 \\
\mathbf{f} \\
\mathbf{b}_{N}
\end{array}\right)
$$

where $A=\operatorname{diag}\left[A_{1}, \ldots, A_{m}\right], B=\operatorname{diag}\left[B_{1}, \ldots, B_{m}\right]$, 


$$
\begin{aligned}
& A_{l}=\left(a_{i k}\right)=\int_{T_{l}} K_{l}^{-1} \boldsymbol{w}_{i}^{(l)} \cdot \boldsymbol{w}_{k}^{(l)} \mathrm{d} \Delta, \quad B_{l}=\left(b_{i}\right)=\int_{T_{l}} \boldsymbol{\nabla} \cdot \boldsymbol{w}_{i}^{(l)} \mathrm{d} \Delta, \\
& Q=\left(q_{r j}\right)=\int_{\partial T_{l}} \mu_{j} \boldsymbol{w}_{i}^{(l)} \cdot \boldsymbol{n}_{i}^{(l)} \mathrm{d} \Gamma, \quad \mathbf{v}=\left(v_{r}\right)=v_{i}^{(l)}, \quad \mathbf{f}=\left(\hat{f}_{l}\right)=\int_{T_{l}} f_{l} \mathrm{~d} \Delta .
\end{aligned}
$$

Here, $i, k=1,2,3,4, j=1, \ldots, n, r=4(l-1)+i$, and $\boldsymbol{\psi}=\left(\psi_{l}\right), \boldsymbol{\lambda}=\left(\lambda_{j}\right)$, and $\mathbf{b}_{N}=\left(b_{N j}\right)$, where $b_{N j}$ is non-zero only if there is a Neumann condition on a boundary face, $e_{j}$. The vector $\boldsymbol{n}_{i}^{(l)}$ is the outward normal to face $i$ of tetrahedron $T_{l}$.

The ensuing system of equations on the unknown $\boldsymbol{\lambda}$ is

$$
Q^{T} M Q \boldsymbol{\lambda}=Q^{T} S H^{-1} \mathbf{f}-\mathbf{b}_{N},
$$

where $H=B^{T} A^{-1} B, S=A^{-1} B$, and $M=A^{-1}-S H^{-1} S^{T}$. The system is symmetric, positive definite, hence it can be efficiently solved by the Preconditioned Conjugate Gradient method. Once $\boldsymbol{\lambda}$ is computed, $\boldsymbol{\psi}$ and $\boldsymbol{v}$ are easily obtained. As opposed to the FE approach, the velocity field thus calculated is inherently mass conserving. The number of faces in the $3 \mathrm{D}$ mesh is also the size of system (2). Note that the coefficient matrix counts no more than five non-zero entries per row.

In the case of MHFE, the number of unknowns is larger than for the $\mathrm{FE}$ scheme, as the number of faces is approximately 6 times that one of nodes. On the other hand, the MHFE system matrix is sparser than the corresponding FE matrix, yielding a relatively more efficient matrix-vector product. However, the computational cost of the MHFE linear system solution is always higher than that of FE.

\section{$2 \quad$ Numerical Results}

\section{$2.1 \quad$ Example 1}

The first example considers a cubic domain $\Omega=[0,1] \times[0,1] \times[0,1]$. The discretization is obtained as follows. The coarsest mesh level $(\ell=1)$ is obtained by subdividing the topmost horizontal face of the cube into $8 \times 8$ squares, cut along the diagonal to form a mesh of 128 triangles. This surface mesh is then replicated vertically (along $z$ ) to form 8 layers. Each triangle is thus the base of a rectangular prism that is subdivided into 3 tetrahedrons by suitably taking one main diagonal over each one of its vertical faces 8 . The mesh thus obtained counts 729 nodes, 3072 tetrahedrons, and 6528 faces. The next coarser mesh levels, $\ell=2,3$, are obtained by uniformly refining the previous level surface mesh (each triangle is subdivided into four triangles by connecting the midpoints of each edge) and by doubling the previous level layers. The second level $(\ell=2)$ is characterized by 4913 nodes, 24576 tetrahedrons, and 50688 faces, while the finest $(\ell=3)$ level has 35937 nodes, 196608 tetrahedrons, and 399360 faces. This procedure guarantees that no tetrahedron face has angles larger than $\pi / 2$. 
We prescribe the analytical solution:

$$
\psi=\cos \left[3 \pi\left(\frac{x^{3}}{3}+\frac{y^{3}}{3}+\frac{z^{3}}{3}-\frac{x^{2}}{2}-\frac{y^{2}}{2}-\frac{z^{2}}{2}\right)\right]
$$

We calculate the related forcing function $f$ in (1), and the Dirichlet boundary conditions. The numerical convergence behavior of our schemes is analyzed by computing suitable relative errors of the pressure and velocity fields on each grid level.

The $L_{2}$ error norm for pressure head is

$$
\left|e_{\ell, \psi}\right|=\left(\sum_{j}\left(\psi\left(\boldsymbol{P}_{j}\right)-\psi_{j}\right)^{2}\right)^{1 / 2} /\left(\sum_{j} \psi\left(\boldsymbol{P}_{j}\right)^{2}\right)^{1 / 2}
$$

When applying the MHFE method, the quantity $\psi\left(\boldsymbol{P}_{j}\right)$ is the analytical pressure at the centroid of the tetrahedron $T_{j}$. When applying the FE scheme, $\psi\left(\boldsymbol{P}_{j}\right)$ is the analytical pressure on the node $\boldsymbol{P}_{j}$. The value $\psi_{j}$ is the numerical approximation to each of these values, obtained by MHFE and FE methods, respectively.

Our measure of the $L_{2}$ error on the velocity is

$$
\left|e_{\ell, \boldsymbol{v}}\right|=\left(\sum_{j} \sum_{\delta \in\{x, y, z\}}\left(v_{\delta}\left(\boldsymbol{P}_{j}\right)-v_{\delta, j}\right)^{2}\right)^{1 / 2} /\left(\sum_{j} \sum_{\delta \in\{x, y, z\}} v_{\delta}\left(\boldsymbol{P}_{j}\right)^{2}\right)^{1 / 2}
$$

Here, $\left(v_{x}\left(\boldsymbol{P}_{j}\right), v_{y}\left(\boldsymbol{P}_{j}\right), v_{z}\left(\boldsymbol{P}_{j}\right)\right)$ is the analytical velocity on the centroid of each element, while $\left(v_{x, j}, v_{y, j}, v_{z, j}\right)$ is the corresponding numerically approximated vector.

Table 1. Numerical convergence behavior of MHFE and FE methods.

\begin{tabular}{|c||c|c|c|c||c|c|c|c|}
\hline \multicolumn{1}{|l||}{$\ell$} & \multicolumn{4}{c||}{ MHFE } & \multicolumn{4}{c|}{$\mathrm{FE}$} \\
\cline { 2 - 9 } & $\left|e_{\ell, \psi}\right|$ & $p$ & $\left|e_{\ell, \boldsymbol{v}}\right|$ & $p$ & $\left|e_{\ell, \psi}\right|$ & $p$ & $\left|e_{\ell, \boldsymbol{v}}\right|$ & $p$ \\
\hline 1 & $1.29 \mathrm{e}-2$ & & $9.00 \mathrm{e}-2$ & & $6.60 \mathrm{e}-3$ & & $1.23 \mathrm{e}-1$ & \\
2 & $3.38 \mathrm{e}-3$ & 1.93 & $4.50 \mathrm{e}-2$ & 1.00 & $1.79 \mathrm{e}-3$ & 1.88 & $6.14 \mathrm{e}-2$ & 1.00 \\
3 & $8.58 \mathrm{e}-4$ & 1.98 & $2.25 \mathrm{e}-2$ & 1.00 & $4.67 \mathrm{e}-4$ & 1.94 & $3.07 \mathrm{e}-2$ & 1.00 \\
\hline
\end{tabular}

Table 1 shows the numerical convergence orders, $p=\log _{2}\left(\left|e_{\ell, \delta}\right| /\left|e_{\ell-1, \delta}\right|\right)$, where either $\delta=\psi$, or $\delta=\boldsymbol{v}$. The numerical order well compares with the theoretical values, which are $p=2$ for the head, $p=1$ for the velocity, showing that asymptotic conditions hold for our domain discretizations.

MHFE mass balance errors are under $10^{-10}$ (the tolerance for the linear system solution), while the FE errors range between $10^{-5}$ and $10^{-10}$. 


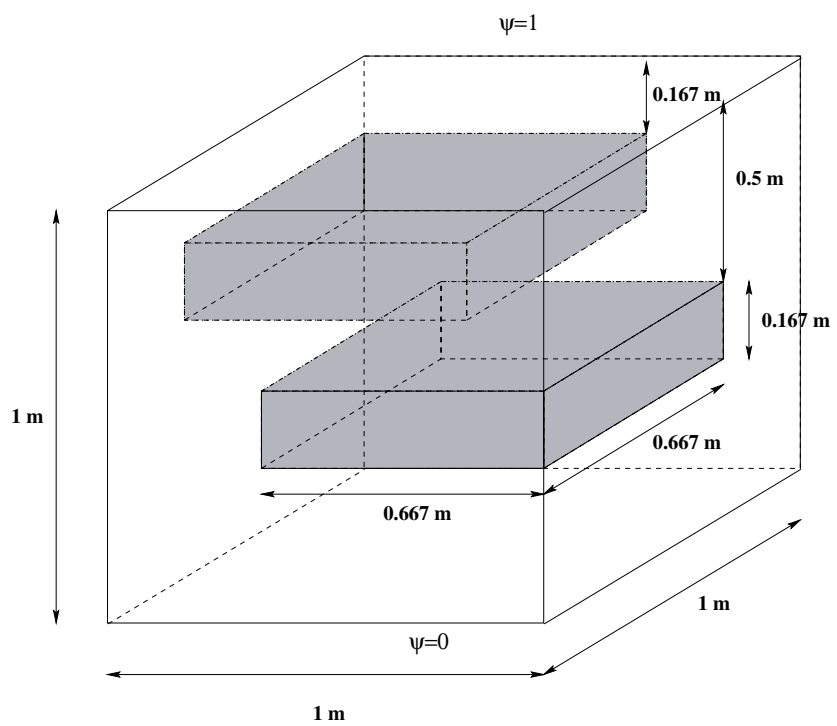

Fig. 1. Domain of the heterogeneous example with Dirichlet boundary conditions.

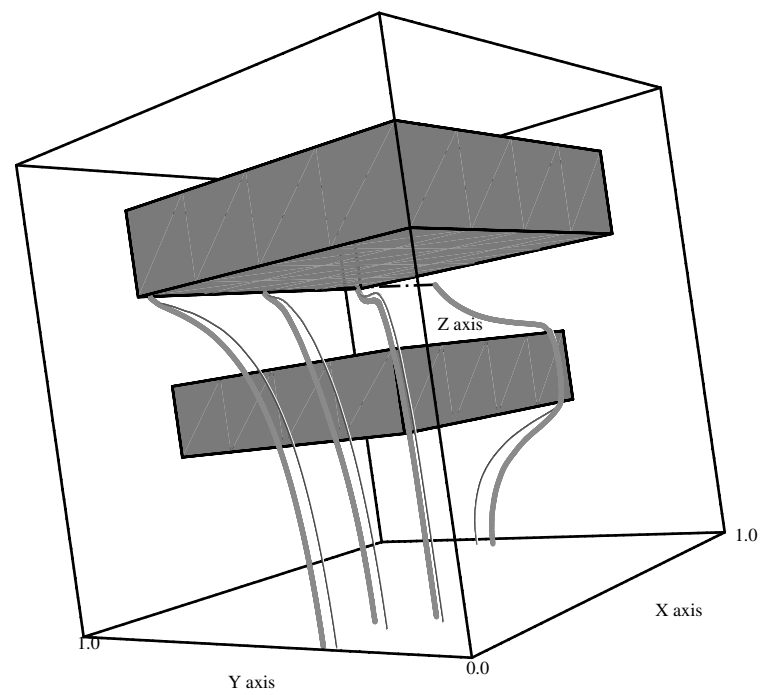

Fig. 2. Three-dimensional view of the calculated streamlines at mesh level $\ell=4$. Thick gray lines show the FE streamlines, thin lines correspond to MHFE ones. 

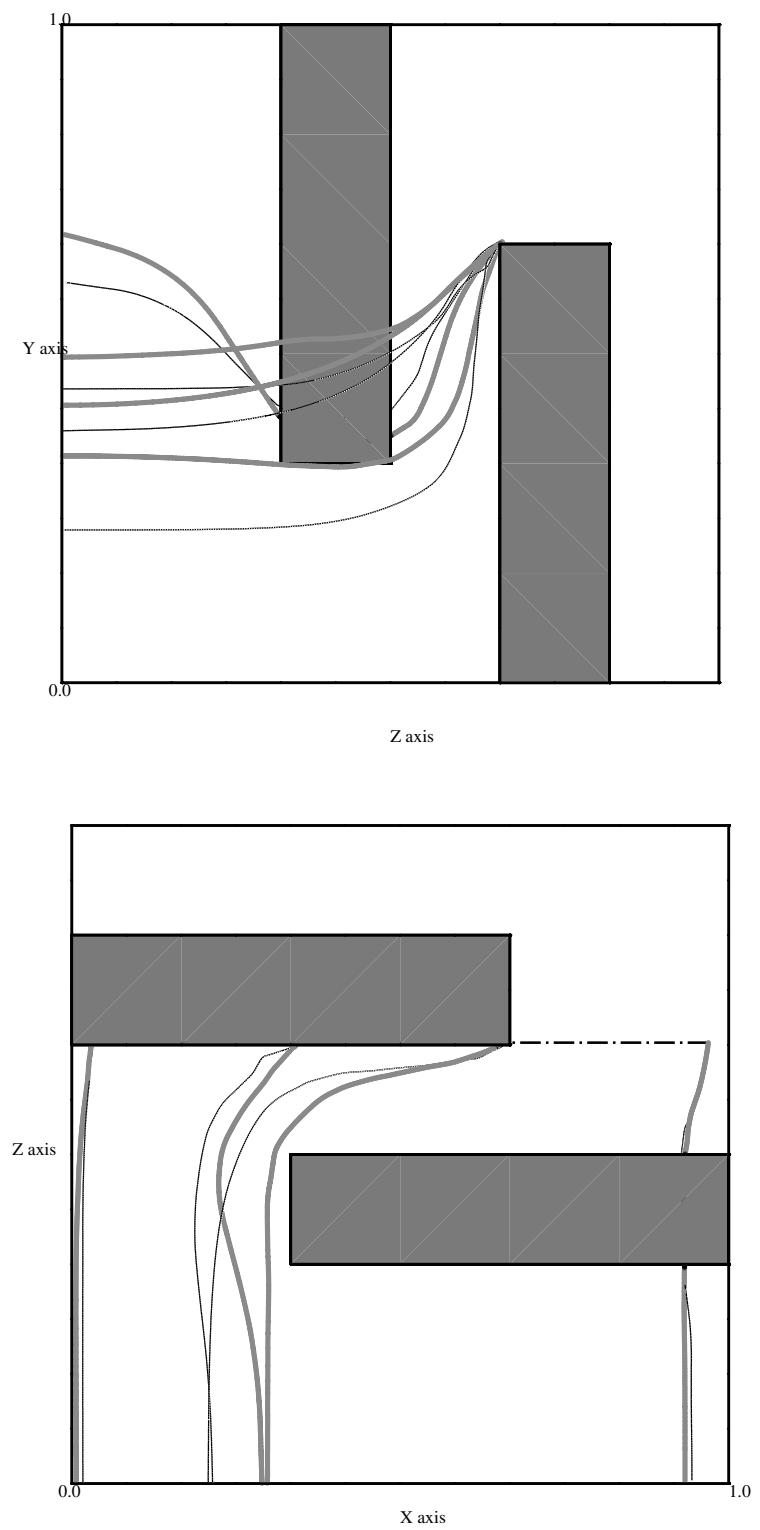

Fig. 3. Side (top) and front (bottom) projections of the calculated streamlines at mesh level $\ell=2$. Thick gray lines show the FE streamlines, thin lines correspond to MHFE ones.

\subsection{Heterogeneous Example}

In this test case we consider a heterogeneous flow problem. Figure 1 sketches the domain, which is again the unit cube with uniform background permeability 

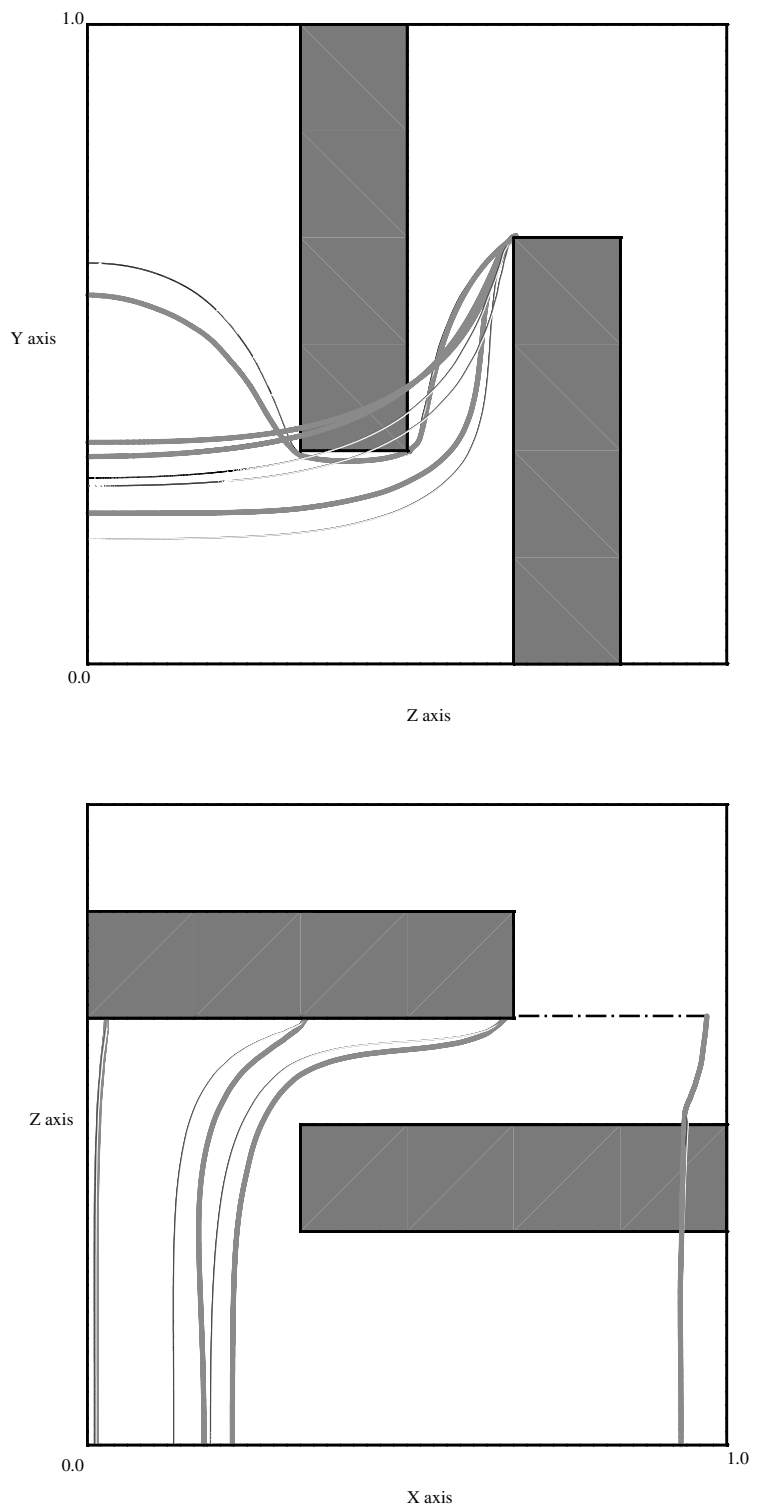

Fig. 4. The same as the previous Figure, for mesh level $\ell=4$.

$\boldsymbol{K}_{x}=\boldsymbol{K}_{y}=\boldsymbol{K}_{z}=1$. Two blocks at lower permeability, $\boldsymbol{K}_{x}=\boldsymbol{K}_{y}=\boldsymbol{K}_{z}=$ $10^{-3}$, have been inserted (the shaded volumes in the Figure). Dirichlet boundary conditions, $\psi=1$ and $\psi=0$, are imposed on the top and bottom face of the domain, respectively. All the vertical faces have a no flow Neumann boundary 
condition. The domain is discretized using the same procedure as before. Four mesh levels $\ell=1,2,3,4$ are considered.

No theoretical solution is available for this test problem, and thus we do not carry out convergence tests. Instead we look at streamlines (Figure 2) calculated from the velocity field. We consider a horizontal line passing through the edge of the uppermost block (shown in Figure 2 as dash-dotted line) and draw the streamlines that start from four evenly spaced points on this line. The streamlines are computed using an adaptive Runge-Kutta method of order 4. Figure 3 shows the trace of the streamlines projected on the $y-z$ plane (top), and the projection on the $x-z$ plane (bottom), for the solution at level $\ell=2$. Figure 4 shows the same results for $\ell=4$. The thick gray lines correspond to the FE solution, while the thin lines correspond to the MHFE solution.

It is interesting to note the strong differences between the FE and MHFE streamlines, which hold even in the finest mesh. Note also that the MHFE streamlines change from level to level less than the corresponding FE streamlines, showing that MHFE may be more accurate then FE in this respect. However, both FE and MHFE display large deviations between the $\ell=2$ and $\ell=4$ cases.

Acknowledgments. This work has been partially supported by Italian MURST Project "Mathematical Models and Numerical Methods for Environmental Fluid Dynamics".

\section{References}

1. Forsyth, P.A.: A control volume finite element approach to NAPL groundwater contamination. SIAM J. Sci. Stat. Comput. 12 (1991) 1029-1057

2. Putti, M., Cordes, C.: Finite element approximation of the diffusion operator on tetrahedra. SIAM J. Sci. Comput. 19 (1998) 1154-1168

3. Cordes, C., Putti, M.: Accuracy of Galerkin finite elements for the groundwater flow equation in two and three dimensional triangulations. Int. J. Numer. Methods Engrg. 52 (2001) 371-387

4. Mazzia, A.: Mixed Finite Elements and Finite Volumes for the solution of density dependent flow and transport of radioactive contaminants in porous media. $\mathrm{PhD}$ thesis, Università di Padova (1999)

5. Mazzia, A., Bergamaschi, L., Putti, M.: A time-splitting technique for advectiondispersion equation in groundwater. J. Comp. Phys. 157 (2000) 181-198

6. Raviart, P.A., Thomas, J.M.: A mixed finite element method for second order elliptic problems. In Galligani, I., Magenes, E., eds.: Mathematical Aspects of the Finite Elements Method. Number 606 in Lecture Notes in Mathematics, New York, Springer-Verlag (1977)

7. Brezzi, F., Fortin, M.: Mixed and Hybrid Finite Element Methods. Springer-Verlag, Berlin (1991)

8. Gambolati, G., Pini, G., Tucciarelli, T.: A 3-D finite element conjugate gradient model of subsurface flow with automatic mesh generation. Adv. Water Resources 3 (1986) 34-41 